\title{
Dynamics of propane in Na-Y zeolite
}

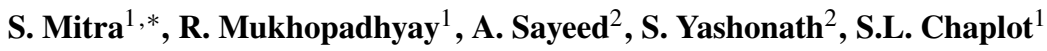 \\ ${ }^{1}$ Solid State Physics Division, Bhabha Atomic Research Centre, Trombay, Mumbai 400 085, India \\ ${ }^{2}$ Solid State and Structural Chemistry unit, Indian Institute of Science, Bangalore 560 012, India \\ Received: 11 July 2001/Accepted: 24 October 2001 - (C) Springer-Verlag 2002
}

\begin{abstract}
The dynamics of propane confined in Na-Y zeolite was studied using the quasi-elastic neutron scattering technique at different temperatures. The data were analysed using a model in which the propane molecules undergo randomwalk diffusion characterised by a Gaussian distribution of jump lengths inside zeolite cages. The diffusion constant and root-mean-square jump length were determined in the temperature range of 300-350 K.
\end{abstract}

PACS: 61.12.Ex; 81.05.Rm

Zeolites are porous aluminosilicate crystals that present some structural cavities regularly distributed over the crystal lattice. These cavities are connected through pores or windows whose diameters depend on the nature of the zeolite. These materials offer an interesting tool for physicists, as the cavities may be utilized to isolate one or several molecules, and thus constitute a simple physical model. A better understanding of the molecular diffusion in zeolite is of great theoretical and technological interest. Technological interest in zeolites arises from the fact that they are widely used in industrial processes as catalysts and molecular sieves, where diffusion of various molecules inside these porous materials plays a crucial role. The molecular sieving property of zeolites is used in the separation of mixtures of hydrocarbons [1]. For these reasons, diffusion of hydrocarbons in zeolites has been studied extensively with various experimental techniques as well as computer simulations. Macroscopic transport diffusion is measured using techniques such as gravimetry, volumetry, chromatography, etc. Self-diffusion coefficients related to the diffusivity on a microscopic scale are measured using two techniques - pulsed field gradient nuclear magnetic resonance (PFG-NMR) [2] and quasi-elastic neutron scattering (QENS) [3, 4]. PFG-NMR and QENS have been observed to give results that are comparable to each other and usually in good agreement with simulation results [1].

*Corresponding author.

(Fax: +91-22/5505151, E-mail: smitra@apsara.barc.ernet.in)
Here we present the results of QENS measurements that we have carried out to study the diffusion of propane gas in Na-Y zeolite. Preliminary studies have been presented elsewhere [5]. The Na-Y structure is made up of a network of tetrahedrally connected pores ( $\alpha$-cages) of diameter $\sim 11.8 \AA$. The pores are interconnected through windows of aperture $\sim 8 \AA$. Propane ("size" $\sim 6 \AA$ ) is one of the simplest hydrocarbons in the alkane series.

\section{Experiment}

The Na-Y zeolite $\left(\mathrm{SiO}_{2} / \mathrm{Al}_{2} \mathrm{O}_{3}\right.$ ratio (wt. \%) $=2.5$, as determined from chemical analysis) used for the experiment was first thoroughly dehydrated through evacuation to $10^{-4} \mathrm{~Pa}$ at a temperature of $400{ }^{\circ} \mathrm{C}$ for $12 \mathrm{~h}$. It was then transferred into a slab-shaped aluminum container of circular geometry. The zeolite sample was loaded with propane gas (purity $>99.9 \%$ ) to saturation, which amounts to about 4.5 molecules per zeolitic supercage [6]. The QENS experiments were performed using the quasi-elastic spectrometer [7] at the Dhruva reactor at Trombay. The instrument is based on a double monochromator for variation of incident energy and an air-cushionbased spectrometer for energy analysis. A double monochromator (pyrolitic graphite crystals) placed inside the pile block of the reactor provides a closer approach to the source, and we have used vertically bent crystals to focus the neutrons at the sample position and thereby enhance the neutron intensity. The spectrometer is used in multi-angle reflecting X-tal (MARX) mode, where one essentially uses a combination of a large crystal analyser and a linear position-sensitive detector, providing larger throughput. This is essential for such experiments to be carried out in a medium flux reactor. A pyrolitic graphite crystal is used as an analyser as well. In the present configuration the spectrometer has an energy resolution of $200 \mu \mathrm{eV}$ with an incident energy of $5.1 \mathrm{meV}$ covering an energy transfer range of $\pm 1 \mathrm{meV}$. The Quasi-elastic spectra were recorded in the wavevector transfer $(Q)$ range of 0.67 to $1.8 \AA^{-1}$ at 300,323 and $350 \mathrm{~K}$ for both bare zeolite and that loaded with propane samples. Spectra on the bare zeolite sample was recorded in order to estimate the contri- 
bution of bare zeolite to the total spectra. The quantity of the propane present in the sample was independently estimated also from a neutron transmission measurement and found to be in close agreement with the loading amount.

\section{Results and discussion}

The spectra from bare zeolite did not show any quasi-elastic broadening at any $Q$-value within the range covered in the present experiment. However, the sample loaded with propane (full saturation) showed quasi-elastic broadening at all the $Q$-values, suggesting the presence of dynamical motion related to the propane adsorbed inside the zeolite cages. Measurements at higher temperatures showed an enhanced quasi-elastic component. The typical QENS spectra at room temperature and at different $Q$-values are shown in Fig. 1. The effect of an increase in temperature at a typical $Q$-value of $1.32 \AA^{-1}$ is shown in Fig. 2 .

In a neutron scattering experiment the measured intensity is proportional to the double-differential cross-section, and for a hydrogenous sample we can write [4]

$\frac{\partial^{2} \sigma}{\partial \omega \partial \Omega} \propto \frac{k}{k_{0}}\left[\sigma_{\mathrm{inc}} S_{\mathrm{inc}}(Q, \omega)\right]$.

$S_{\text {inc }}(Q, \omega)$ is known as the scattering law, and "inc" stands for incoherent part. $\boldsymbol{k}$ and $\boldsymbol{k}_{0}$ are the initial and final wavevectors and $Q=\left|\boldsymbol{k}-\boldsymbol{k}_{0}\right|$.

In principle both rotational and translational motion could contribute to the measured quasi-elastic spectrum. In the first

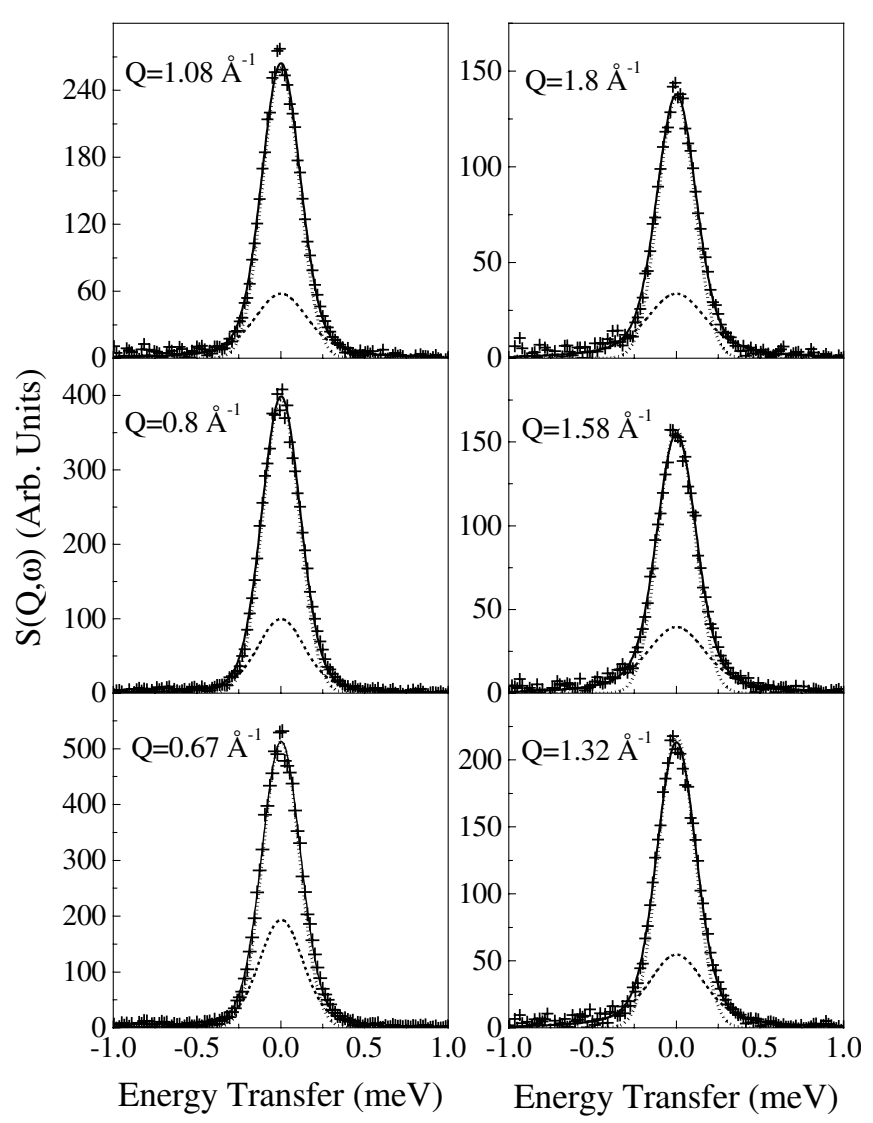

Fig. 1. QENS spectra of propane in $\mathrm{Na}-\mathrm{Y}$ zeolite at $300 \mathrm{~K}$

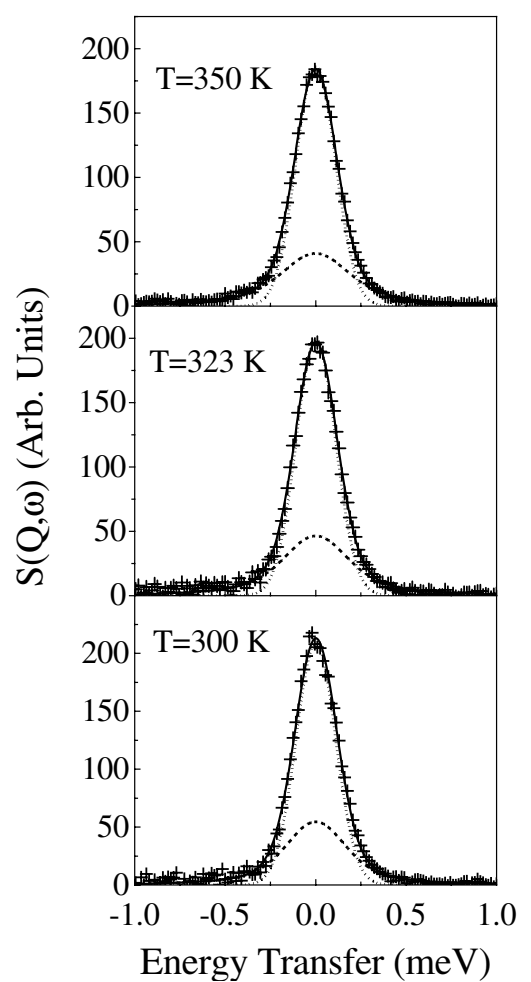

Fig. 2. QENS spectra of propane in Na-Y zeolite at $1.32 \AA^{-1}$ at different temperatures

instance we tried to separate the elastic and quasi-elastic components in the whole spectrum. It was found that there is no extra elastic component other than that which is present in the bare zeolite itself, and only a single Lorenzian could describe the quasi-elastic part, suggesting that the rotation of the propane does not contribute to the present data. Therefore, we assumed that only the translational motion of the propane molecules contributed to the observed dynamics in the present experiment. Analysis of the QENS spectra from the propane molecules was attempted using several models corresponding to the translational motion of the propane molecules, and it was found that a model of $S(Q, \omega)$ proposed by Hall and Ross [8] is adequate and consistent with the experimental data. In this model it is assumed that the particles undergo random translational diffusion with a Gaussian distribution of jump lengths. The scattering law in this case will be a Lorentzian as follows:

$S(Q, \omega)=\frac{1}{\pi} \frac{\Gamma(Q)}{[\Gamma(Q)]^{2}+\omega^{2}}$,

with

$\Gamma(Q)=\frac{1}{\tau}\left[1-\exp \left(-\frac{Q^{2}\left\langle l^{2}\right\rangle}{6}\right)\right]$,

where $\tau$ and $\left\langle l^{2}\right\rangle$ are the residence time and mean square jump length, respectively. The QENS data were collected on both propane loaded samples and dehydrated samples at room temperature. Analysis of the data involved convolution of the scattering law with the instrumental resolution function, and the parameters were obtained by least-squares fitting. A very good fit was obtained for the whole $Q$ range. The typical 


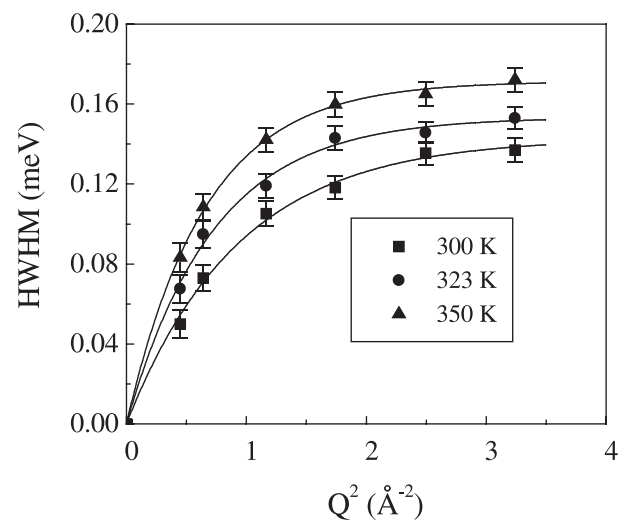

Fig. 3. Variation of the HWHM of the quasi-elastic lines with $Q^{2}$

quality of fit and the separated quasi-elastic components are shown in Fig. 1. Dashed lines in Fig. 1 represent the quasielastic line arising from the diffusion of propane molecules, and dotted lines represent the instrumental resolution function. Similarly the fit at different temperatures for a typical value of $Q=1.32 \AA^{-1}$ is shown in Fig. 2. Figure 3 shows the variation of the HWHM of the Lorentzian lines with $Q^{2}$ as obtained from the least-squares fit of the model $S(Q, \omega)$ (2) with the measured data at different temperatures.

The solid line was obtained by least-squares fitting using (3) with $\tau$ and $\left\langle l^{2}\right\rangle$ as parameters. The values of $\tau$ and $\left\langle l^{2}\right\rangle^{0.5}$ obtained from the fit at different temperatures are given in Table 1. The estimated values of the diffusion constant $D$, where

$$
D=\frac{\left\langle l^{2}\right\rangle}{6 \tau},
$$

are also given in the table. The activation energy of the jump diffusion process has been found to be $3.6( \pm 0.5) \mathrm{kJ} \mathrm{mol}^{-1}$. The values of the diffusion constant obtained here were found to be higher than the previous NMR measurements of Caro et al. [3] in Na-X, a similar system. However, this is expected, considering the difference in $\mathrm{Si} / \mathrm{Al}$ ratios of the $\mathrm{Na}-\mathrm{X}$ and $\mathrm{Na}-\mathrm{Y}$ zeolite samples. The variations in residence time and mean jump length with temperature are consistent with earlier studies $[9,10]$.
Table 1. Values of the residence time, mean jump length and diffusion constant of propane in Na-Y zeolite at different temperatures

\begin{tabular}{cccc}
\hline$T(\mathrm{~K})$ & $\tau(\mathrm{ps})$ & $\left\langle l^{2}\right\rangle^{0.5}(\AA)$ & $D\left(\times 10^{-5} \mathrm{~cm}^{2} \mathrm{~s}^{-1}\right)$ \\
\hline 300 & $4.6 \pm 0.5$ & $2.5 \pm 0.2$ & $2.3 \pm 0.3$ \\
323 & $4.3 \pm 0.4$ & $2.9 \pm 0.2$ & $3.2 \pm 0.3$ \\
350 & $3.8 \pm 0.3$ & $3.0 \pm 0.3$ & $4.0 \pm 0.4$ \\
\hline
\end{tabular}

It may be further noted that preliminary results of our recent calculation using molecular dynamics simulation show very good agreement with the present QENS data.

\section{Conclusion}

The results of QENS measurements carried out to study the diffusion of propane gas in zeolite $\mathrm{Na}-\mathrm{Y}$ at different temperatures have been reported. The experimental data were found to be consistent with a model in which the propane molecules undergo random diffusion with Gaussian-distributed jump lengths inside the zeolite cages. The present results are consistent with PFG-NMR results for the diffusion of propane in zeolite Na-X, a similar system. The variations in $\tau$ and $\left\langle l^{2}\right\rangle$ with temperature are also consistent with the earlier studied systems.

\section{References}

1. S.P. Bates, R.A. van Santen: Adv. Catal. 42, 1 (1998)

2. J. Kärger, D.M. Ruthven: Diffusion in Zeolites (Wiley, New York 1992)

3. J. Caro, M. Bülow, W. Schimer, J. Kärger, W. Heink, H. Pfeifer, S.P. Zdanov: J. Chem. Soc., Faraday Trans. 1 81, 2541 (1985)

4. M. Bée: Quasielastic Neutron Scattering (Adam Hilger, Bristol 1988)

5. A. Sayeed, S. Mitra, R. Mukhopadhyay, S. Yashonath, S.L. Chaplot: Proc. Solid State Phys. 42, 352 (1999)

6. J. Kärger, H. Pfeifer, M. Rauscher, A. Walter: J. Chem. Soc., Faraday Trans. 1 76, 717 (1980)

7. R. Mukhopadhyay, S. Mitra, S.K. Paranjpe, B.A. Dasannacharya: Nucl. Instrum. Methods A 474, 55 (2001)

8. P.L. Hall, D.K. Ross: Mol. Phys. 42, 673 (1981)

9. H. Jobic, M. Bée, J. Caro, M. Büllow, J. Kärger: J. Chem. Soc., Faraday Trans. $1 \mathbf{8 5}, 4201$ (1989)

10. H. Jobic, M. Bée, G.J. Kearley: Zeolites 9, 312 (1989) 